\title{
Author's Reply: Emergency Department Versus Operating Suite Intubation in Operative Trauma Patients: Does Location Matter?
}

\author{
Ryan P. Dumas ${ }^{1} \cdot$ Mark J. Seamon ${ }^{2}$
}

Published online: 26 April 2020

(c) Société Internationale de Chirurgie 2020

We would like to address Dr. Elkbuli's letter regarding our manuscript entitled: "Emergency Department Versus Operating Suite Intubation in Operative Trauma Patients: Does Location Matter?" Dr. Elkbuli and colleagues raise some important points about our work, and we appreciate their time and commentary. We believe our manuscript, and this response addresses many of the concerns raised. Given that our study was retrospective in nature, the only consistent and complete data regarding patient physiology were vitals on arrival. The authors completely agree that the physiology of traumatically injured patients may change very rapidly and that what is true about arrival physiology may not remain true immediately pre-intubation. The authors acknowledge that this is an important limitation of this work. Frequently, given acuity of these patients, abnormal vitals may not be documented at all, or may be incompletely documented in the medical record making immediate pre-intubation vitals difficult to capture retrospectively. A prospective trial designed to capture immediate pre-intubation vitals would be needed to overcome this shortcoming.

While abdominal trauma may be the most studied, regardless of location, time to hemorrhage control has been shown throughout the literature to impact outcomes. By choosing an ED dwell time of less than $30 \mathrm{~min}$, we identified patients in need of immediate hemorrhage control. We believe that time to hemorrhage control is more

Ryan P. Dumas

ryan.dumas@utsouthwestern.edu

1 Division of General and Acute Care Surgery, University of Texas Southwestern Medical Center, Dallas, TX, USA

2 Division of Traumatology, Surgical Critical Care and Emergency Surgery, University of Pennsylvania, Philadelphia, PA, USA important than location of hemorrhage. Additionally, some of the original literature regarding prompt surgical management by Hoyt et al. [1] analyzed both chest and abdominal trauma victims. These authors also believe that significant injury patterns such as traumatic pericardial tamponade may be best addressed with delayed intubation in the operating suite which is why these procedures were included in this analysis.

Finally, with regard to the statistical analysis, the authors were only able to analyze total elapsed time as a continuous linear variable given the small number of cardiac arrests in the cohort $(n=12)$. A logistic regression on the binary outcome of cardiac arrest, as these authors suggest, cannot be performed on the cardiac arrest data as there are too few arrest victims. We are unable to create a multivariate model because the outcome of interest is too infrequent. In order to account for all arrival physiology variables, we would need approximately 50-60 cardiac arrest patients to adhere to principles that apply when constructing a statistical model. Such an analysis could conceivably be performed on a much larger dataset.

\section{Reference}

1. Hoyt DB, Shackford SR, McGill T et al (1989) The impact of inhouse surgeons and operating room resuscitation on outcome of traumatic injuries. Arch Surg 124:906-909 (discussion 909-910)

Publisher's Note Springer Nature remains neutral with regard to jurisdictional claims in published maps and institutional affiliations. 